\title{
COMPLICAÇÕES NO PÓS-OPERATÓRIO IMEDIATO DE REVASCULARIZAÇÃO DO MIOCÁRDIO
}

\author{
Immediate postoperative complications of myocardio revascularization
}

\author{
Complicaciones en el postoperatorio inmediato de revascularización miocárdica \\ Alessandra Yuri Takehana de Andrade ${ }^{1 *}$ (D) Patricia Sayuri de Lima Tanaka² (D), \\ Vanessa de Brito Poveda ${ }^{3}$ (D) Ruth Natalia Teresa Turrini ${ }^{4}$
}

RESUM0: Objetivo: Verificar as principais complicações da cirurgia de revascularização do miocárdio (RM) com circulação extracorpórea (CEC) e sua associação com os fatores de risco modificáveis e não modificáveis, diagnósticos de enfermagem, tempo de CEC e carga horária de enfermagem. Método: Coorte retrospectiva por meio da análise de 50 prontuários de pacientes adultos submetidos à RM com CEC, entre 2012 e 2017. Projeto aprovado pelo Comitê de Ética em Pesquisa parecer $n^{\circ}$ 1969823. Resultados: A amostra foi predominantemente masculina (70\%), com idade média de 62,1 anos (desvio padrão — $\mathrm{DP} \pm 9,98)$. O tempo médio de CEC foi de 124 minutos (DP $\pm 0,46)$. Complicações pós-operatórias ocorreram em $52 \%$ dos 50 pacientes analisados, sendo a mais frequente o sangramento (16\%). O diagnóstico de enfermagem mais frequente foi risco de infecção (92\%). Constatou-se que os cuidados pela equipe de enfermagem foram mais requeridos por pacientes que apresentaram hipotensão $(\mathrm{p}=0,003)$ e arritmia $(\mathrm{p}=0,000)$ no pós-operatório. Conclusão: $\mathrm{O}$ conhecimento das complicações pós-operatórias associadas ao perfil dos pacientes atendidos colabora para a elaboração de planos de cuidados mais adequados. Palavras-chave: Enfermagem. Cirurgia torácica. Cuidados de enfermagem.

ABSTRACT: Objective: To verify the main complications of myocardial revascularization (MR) with cardiopulmonary bypass (CPB) and its association with modifiable and non-modifiable risk factors, nursing diagnoses, CPB time and nursing workload. Method: Retrospective cohort through analysis of 50 medical records of adult patients undergoing MR with CPB between 2012 and 2017. Project approved by the Research Ethics Committee Report No. 1969823. Results: The sample was predominantly male (70\%), with a mean age of 62.1 years (standard deviation - SD \pm 9.98 ). The mean CPB time was 124 minutes ( $\mathrm{SD} \pm 0.46$ ). Postoperative complications occurred in $52 \%$ of the 50 patients analyzed, with bleeding being the most frequent (16\%). The most frequent nursing diagnosis was risk of infection $(92 \%)$. It was found that nursing staff care was most needed by patients with postoperative hypotension $(\mathrm{p}=0.003)$ and arrhythmia $(\mathrm{p}=0.000$ ). Conclusion: Knowledge of postoperative complications associated with the patients helps develop more appropriate care plans. Keywords: Nursing. Thoracic surgery. Nursing care.

RESUMEN: Objetivo: Verificar las principales complicaciones de la cirugía de revascularización miocárdica (RM) con bypass cardiopulmonar (BCP) y su asociación con factores de riesgo modificables y no modificables, diagnósticos de enfermería, tiempo de BCP y carga de trabajo de enfermería. Método: Cohorte retrospectiva mediante el análisis de 50 registros médicos de pacientes adultos sometidos a RM con BCP entre 2012 y 2017. Proyecto aprobado por el Comité de Ética de Investigación Opinión 1969823. Resultados: La muestra fue predominantemente masculina (70\%), con edad media de 62,1 años (DE $\pm 9,98$ ). El tiempo medio de BCP fue de 124 minutos (DE $\pm 0,46$ ). Las complicaciones postoperatorias ocurrieron en el $52 \%$ de los 50 pacientes analizados, siendo el sangrado el más frecuente (16\%). El diagnóstico de enfermería más frecuente fue el riesgo de infección (92\%). Se encontró que la atención por parte del personal de enfermería era más necesaria para los pacientes que presentaban hipotensión ( $\mathrm{p}=0,003)$ y arritmia $(\mathrm{p}=0,000)$ después de la operación. Conclusión: El conocimiento de las complicaciones postoperatorias asociadas con el perfil de los pacientes ayudó en la elaboración de planes de atención más apropriados. Palabras clave: Enfermería. Cirugía torácica. Atención de enfermería.

'Enfermeira responsável técnica da Pró-Saúde Associação Beneficente Assistência Social e Hospitalar (ABASH) do Centro de Atenção Psicossocial em Álcool e outras Drogas (CAPS AD). Graduada em Enfermagem pela Escola de Enfermagem da Universidade de São Paulo (USP) - São Paulo (SP), Brasil.

Enfermeira; especialista em Auditoria pelo Instituto Brasileiro de Extensão Educacional. Enfermeira assistencial da Rede Sarah de Hospitais de Reabilitação Associação das Pioneiras Sociais - Brasília (DF), Brasil. '2Enfermeira; especialista em Auditoria pelo Instituto Brasileiro de Extensão Educacional. Enfermeira assistencial da Rede Sarah de Hospitais de Reabilitação Associação das Pioneiras Sociais - Brasilia (DF), Brasil. ¿Doutora em Saúde Pública pela Faculdade de Saúde Pública da USP. Professora associada do Departamento de Enfermagem Médico-Cirúrgica da Escola de Enfermagem da USP - São Paulo (SP), Brasil. *Autora correspondente: alessandra.yuri.andrade@usp.br

Recebido: 04/09/2018 - Aprovado: 15/09/2019

DOI: $10.5327 / Z 1414-4425201900040008$ 


\section{INTRODUÇÃO}

Atualmente, as doenças crônicas não transmissíveis (DCNT) são responsáveis por altas taxas de morbimortalidade no país ${ }^{1}$. Entre elas, destacam-se as doenças cardiovasculares ${ }^{2}$. A ocorrência das doenças cardiovasculares está relacionada a diversos fatores considerados de risco para o adoecimento causado por esses agravos, tais quais hábitos de vida prejudiciais, como o tabagismo, a alimentação inadequada, o sedentarismo e o consumo de álcool ${ }^{3}$.

Assim, o tratamento dessas afecções cardíacas pode ocorrer tanto por meio farmacológico, via cateterismo percutâneo, quanto cirúrgico, pela revascularização do miocárdio $(\mathrm{RM})^{4}$.

A RM é frequentemente realizada mediante a circulação extracorpórea (CEC). Essa técnica aplicada às cirurgias cardíacas permitiu um campo cirúrgico limpo e seguro à equipe, preservando as características funcionais do aparelho cardíaco 5 .

Contudo, apesar dos benefícios ocasionados pelo uso da CEC, sua utilização também pode estar relacionada a potenciais complicações pós-operatórias imediatas (POI), especialmente durante períodos prolongados, em pacientes idosos $\mathrm{e}$ em bebês menores de 3 meses $^{5}$.

As complicações relacionadas ao uso da CEC estão associadas à indução da resposta inflamatória sistêmica orgânica, com prejuízo da coagulação e da resposta imune; aumento do tônus venoso; aumento da liberação de catecolaminas; alterações no estado eletrolítico; disfunção, lesão ou necrose celular do miocárdio; e disfunção pulmonar branda, o que acarreta complicações nos sistemas cardiovascular, respiratório, renal, gastrointestinal e nervoso ${ }^{5}$.

Considerando que o enfermeiro é um dos principais agentes do cuidado, é necessário que ele esteja alerta aos riscos e problemas que acometem os pacientes sob seus cuidados, para que preste assistência de qualidade e, sempre que possível, livre de riscos ${ }^{4}$. Desde o levantamento do histórico de enfermagem até o tratamento das possíveis complicações pós-operatórias, é importante conhecer como esses antecedentes pessoais estão relacionados às principais complicações no POI de pacientes que passaram pelo procedimento de RM com CEC.

Nesse sentido, a aplicação dos diagnósticos de enfermagem é imprescindível, pois permite o direcionamento efetivo da assistência para as necessidades individuais de cada paciente, por meio da escolha da intervenção ideal, permitindo sua subsequente avaliação.

\section{OBJETIVO}

Verificar as principais complicações da RMcom CEC e sua associação com os fatores de risco modificáveis e não modificáveis, os diagnósticos de enfermagem, o tempo de circulação extracorpórea e a carga horária de enfermagem.

\section{MÉTODO}

Estudo descritivo-exploratório e retrospectivo desenvolvido por meio da análise de prontuários de pacientes submetidos a cirurgias cardíacas realizadas entre 2012 e 2017.

Para tanto, foi utilizada uma amostra por conveniência composta de 50 prontuários de pacientes maiores de 18 anos submetidos à cirurgia de RM com CEC e sobreviventes às primeiras 72 horas de pós-operatório. Foram excluídos os pacientes submetidos a outros procedimentos cirúrgicos de qualquer natureza, realizados nos 30 dias antecedentes ao procedimento de RM, os submetidos a tratamento hemodialítico anterior ao procedimento cirúrgico analisado, as cirurgias de RM sem CEC e as cirurgias de urgência e emergência.

A coleta de dados ocorreu por uma das pesquisadoras por meio de um instrumento que continha dados de identificação sociodemográfica, antecedentes pessoais clínicos e cirúrgicos, medicamentos de uso contínuo, sinais vitais, dispositivos invasivos utilizados nos períodos pré e pós-operatório, duração da cirurgia, tempo de CEC, tempo de internação na unidade de terapia intensiva (UTI), complicações cirúrgicas, diagnósticos de enfermagem e número de procedimentos feitos ou auxiliados pela equipe de enfermagem no POI.

A lista dos pacientes submetidos aos procedimentos de RM entre 2012 e 2017 foi fornecida pelo serviço de arquivo médico do hospital selecionado para o estudo. Com base nessa lista e aplicados os critérios de inclusão e exclusão, foi realizada a leitura, na íntegra, dos prontuários.

Para este estudo, foram seguidas as diretrizes e normas regulamentadoras de pesquisas envolvendo seres humanos, emanadas da Resolução $n^{\circ} 466$ de 2012 do Conselho Nacional de Saúde ${ }^{6}$. O projeto foi submetido ao Comitê de Ética em Pesquisa da Escola de Enfermagem da Universidade de São Paulo e recebeu aprovação sob número de protocolo 1969823.

Os resultados foram analisados pelo programa Statistical Package for the Social Sciences (SPSS), por meio dos testes $\chi^{2}$ com correção de Yates ou teste exato de Fisher para as 
variáveis dicotômicas. Para as variáveis contínuas, foi realizado o teste $t$ de Student ou de Mann-Whitney. O nível de significância delimitado foi $\alpha=0,05$.

Para estimativa da carga horária de trabalho despendida pela equipe de enfermagem, foi utilizado o instrumento nursing activities score (NAS), que considera as atividades básicas, os suportes ventilatório, cardiovascular, renal, neurológico e metabólico e as intervenções específicas. Cada item possui uma pontuação, e o escore do paciente é a soma da pontuação de todos os itens. Esse total representa, em porcentagem, quanto tempo de assistência o paciente exigiu nas 24 horas, sendo seu total máximo 176,8\%. De acordo com a definição, 100 pontos NAS equivale a $100 \%$ do tempo de um profissional de enfermagem nas 24 horas, e cada ponto do NAS corresponde a 14,4 minutos ${ }^{7}$.

\section{RESULTADOS}

A amostra foi composta de 50 prontuários de pacientes, com idades variando entre 32 e 77 anos, sendo a média de 62,1 anos $(\mathrm{DP} \pm 9,98)$, a maioria masculina $(70 \%)$, fumante $(20 \%)$ ou ex-fumante $(32 \%)$ e com antecedentes pessoais variados $(100 \%)$ (Tabela 1$)$.

Tabela 1. Variáveis clínicas e sociodemográficas dos pacientes incluídos na investigação.

\begin{tabular}{|l|c|c|}
\hline Variáveis & n & $\%$ \\
\hline Fatores de risco não modificáveis & & \\
\hline Sexo & & \\
\hline Masculino & 35 & 70 \\
\hline Feminino & 15 & 30 \\
\hline Histórico familiar de cardiopatias & 17 & 34 \\
\hline Fatores de risco modificáveis & & \\
\hline Tabagismo & & \\
\hline Fumante & 10 & 20 \\
\hline Ex-fumante & 16 & 32 \\
\hline Etilismo & & \\
\hline Etilista & 2 & 4 \\
\hline Ex-etilista & 2 & 4 \\
\hline Hipertensão arterial sistêmica & 36 & 72 \\
\hline Dislipidemia & 20 & 40 \\
\hline Doença arterial coronariana & 16 & 32 \\
\hline Obesidade & 2 & 4 \\
\hline
\end{tabular}

Entre os 50 pacientes, $70 \%$ faziam uso de medicamentos em casa, destacando-se anti-hipertensivos (56\%), estatinas (46\%), antiplaquetários (38\%) e hipoglicemiantes orais (18\%).

Oito $(16 \%)$ realizaram alguma cirurgia anterior, destacando-se as cirurgias para retirada parcial ou total de órgãos (75\%), ortopédicas (25\%), vasculares (25\%) e abdominais (25\%). Não houve registros de complicações nesses procedimentos.

Quanto aos medicamentos, $20 \%$ receberam droga vasoativa no pré-operatório, principalmente nitroglicerina (12\%) e dobutamina ( $6 \%$ ), e $40 \%$ dos pacientes apresentavam algum dispositivo no pré-operatório, com destaque para cateter vesical de demora (84\%) e acessos venosos periféricos (36\%).

O tempo cirúrgico variou entre 4 e 16 horas, com média de 9 horas e 20 minutos (desvio padrão - DP $\pm 2,52 \mathrm{~h}$ ). O tempo médio de CEC dos procedimentos realizados foi 2 horas e 4 minutos ( $\mathrm{DP} \pm 0,46 \mathrm{~h}$ ), sendo o mínimo e máximo, respectivamente, de 39 minutos e 3 horas e 40 minutos. Em relação ao tempo de pinçamento aórtico, a média foi de 1 hora e 17 minutos ( $\mathrm{DP} \pm 0,41 \mathrm{~h}$ ), com variação entre 25 minutos e 2 horas e 23 minutos.

Durante o procedimento, $20 \%$ dos pacientes receberam concentrado de hemácias e $24 \%$ exibiram intercorrência cirúrgica. A principal intercorrência descrita foi a dificuldade em retirar o paciente de CEC (50\%), seguida de sangramento $(40 \%)$ e instabilidade hemodinâmica $(30 \%)$.

Complicações no POI ocorreram em 26 (52\%) pacientes, mas apenas um $(2 \%)$ necessitou de reabordagem cirúrgica, decorrente de hemorragia, e dois (4\%) evoluíram a óbito, sendo um deles por choque cardiogênico e outro por problemas cardiológicos não exemplificados (Tabela 2).

As mais frequentes complicações pós-operatórias foram cardiológicas, representadas por arritmias (14\%), hipotensão (10\%), fibrilação atrial (6\%), síndrome do baixo débito cardíaco (4\%) e parada cardiorrespiratória (2\%); hematológicas, representadas por sangramento $(8,16 \%)$ e instabilidade hemodinâmica (4\%); e do sistema respiratório, destacando-se a hipoxemia (2\%) e o pneumotórax (2\%).

No pós-operatório houve necessidade de utilização de mais dispositivos invasivos (96\%) e novos dispositivos, entre eles monitorização da pressão arterial invasiva (90\%), fio de marcapasso (80\%), cateter de Swan-Ganz (12\%) e balão intra-aórtico $(6 \%)$.

Na Tabela 3 estão descritos os diagnósticos de enfermagem encontrados, de acordo com a taxonomia da North American Nursing Diagnosis Association (NANDA) ${ }^{8}$.

As complicações pós-operatórias que apresentaram relação estatística significativa com os fatores de risco modificáveis e não modificáveis foram: histórico familiar de cardiopatias, 
Tabela 2. Distribuição das variáveis clínicas e cirúrgicas dos pacientes incluídos na investigação.

\begin{tabular}{|l|c|c|}
\hline Variáveis & $\mathbf{n}$ & $\%$ \\
\hline Estado físico & 1 & 2 \\
\hline ASA 2 & 28 & 49 \\
\hline ASA 3 & 21 & 42 \\
\hline ASA 4 & & \\
\hline Complicações cirúrgicas & & \\
\hline Complicações no intraoperatório & 6 & 12 \\
\hline Dificuldade de retirar o paciente de CEC & 4 & 8 \\
\hline Sangramento & 3 & 3 \\
\hline Instabilidade hemodinâmica & & \\
\hline Complicações no pós-operatório & 17 & 34 \\
\hline Cardíaca & 10 & 20 \\
\hline Hematológica & 2 & 4 \\
\hline Respiratória & 2 & 4 \\
\hline Renal & 2 & 4 \\
\hline Neurológica & 1 & 2 \\
\hline Reabordagem cirúrgica & 2 & 4 \\
\hline Óbito
\end{tabular}

ASA: American Society of Anesthesiologists; CEC: circulação extracorpórea.

Tabela 3. Distribuição dos diagnósticos de enfermagem dos pacientes incluídos na investigação.

\begin{tabular}{|l|c|c|}
\hline Diagnósticos de enfermagem* & n & $\%$ \\
\hline Risco de infecção & 46 & 92 \\
\hline Risco de perfusão tissular cardíaca diminuída & 30 & 60 \\
\hline Débito cardíaco diminuído & 25 & 50 \\
\hline Risco de choque & 18 & 36 \\
\hline Risco de sangramento & 17 & 34 \\
\hline Dor aguda & 15 & 30 \\
\hline Risco de glicemia instável & 11 & 22 \\
\hline Integridade de pele prejudicada & 10 & 20 \\
\hline Ansiedade & 9 & 18 \\
\hline Risco de queda & 8 & 16 \\
\hline Padrão respiratório ineficaz & 6 & 12 \\
\hline Déficit de autocuidado para banho & 5 & 10 \\
\hline Mobilidade física prejudicada & 5 & 10 \\
\hline Perfusão tissular periférica ineficaz & 5 & 10 \\
\hline Risco de constipação & 4 & 8 \\
\hline Nutrição desequilibrada menor que as & 2 & 4 \\
\hline necessidades & *Ónero de diagnósticos de enfermagemémaior do que o número de pacientes, pois cada \\
\hline paciente apresentava vários diagnósticos.
\end{tabular}

correlacionado a complicações cardiológicas ( $\mathrm{p}=0,050)$; e dislipidemia, ligada à integridade de pele prejudicada $(\mathrm{p}=0,029)$.

Houve associação entre tempo de $\operatorname{CEC~}(\mathrm{p}=0,035)$ e tempo de pinçamento aórtico $(\mathrm{p}=0,039)$, com a probabilidade de o paciente exibir sangramento no pós-operatório, e associação entre o tempo de anestesia com o risco de débito cardíaco diminuído $(\mathrm{p}=0,013)$.

O tempo de CEC também é pertinente ao aparecimento de fibrilação atrial no $\mathrm{POI}(\mathrm{p}=0,011)$ e ao diagnóstico risco de sangramento no que tange às complicações hematológicas $(\mathrm{p}=0,025)$ e à ocorrência de sangramento $(\mathrm{p}=0,038)$. A utilização de fio de marcapasso foi associada ao diagnóstico de dor aguda $(\mathrm{p}=0,026)$. As demais correlações estatisticamente significantes identificadas no presente estudo estão descritas no Quadro 1.

Quadro 1. Correlação entre diagnósticos de enfermagem, complicações pós-operatórias e antecedentes pessoais e clínico-cirúrgicos.

\begin{tabular}{|c|c|c|}
\hline $\begin{array}{l}\text { Diagnósticos de } \\
\text { Enfermagem }\end{array}$ & Variáveis & $\mathbf{p}^{*}$ \\
\hline \multirow{4}{*}{$\begin{array}{l}\text { Risco de } \\
\text { sangramento }\end{array}$} & Tempo de CEC & 0,035 \\
\hline & Tempo de pinçamento aórtico & 0,039 \\
\hline & Complicação hematológica & 0,025 \\
\hline & $\begin{array}{l}\text { Sangramento no } \\
\text { pós-operatório imediato }\end{array}$ & 0,038 \\
\hline \multirow{4}{*}{$\begin{array}{l}\text { Integridade da pele } \\
\text { prejudicada }\end{array}$} & Droga vasoativa & 0,029 \\
\hline & Frequência respiratória & 0,038 \\
\hline & Dislipidemia & 0,029 \\
\hline & Cardiopatias & 0,023 \\
\hline \multirow{2}{*}{$\begin{array}{l}\text { Déficit de } \\
\text { autocuidado para } \\
\text { banho }\end{array}$} & Tempo de CEC & 0,028 \\
\hline & Tempo de anestesia & 0,006 \\
\hline \multirow{3}{*}{$\begin{array}{l}\text { Risco de glicemia } \\
\text { instável }\end{array}$} & Diabetes mellitus & 0,004 \\
\hline & ASA 3 & 0,029 \\
\hline & ASA 4 & 0,014 \\
\hline $\begin{array}{l}\text { Débito cardíaco } \\
\text { diminuído }\end{array}$ & Tempo de anestesia & 0,013 \\
\hline Dor aguda & Diabetes mellitus & 0,052 \\
\hline $\begin{array}{l}\text { Padrão respiratório } \\
\text { ineficaz }\end{array}$ & Tempo de internação & 0,032 \\
\hline $\begin{array}{l}\text { Nutrição menor que } \\
\text { as necessidades }\end{array}$ & Angina & 0,043 \\
\hline Confusão aguda & Neoplasias & 0,043 \\
\hline Risco de queda & Pressão arterial diastólica & 0,046 \\
\hline Risco de choque & Pressão arterial diastólica & 0,034 \\
\hline
\end{tabular}

*Teste exato de Fisher; CEC: circulação extracorpórea; ASA: American Society of Anesthesiologists. 
Quanto à média da carga de trabalho despendida pela equipe de enfermagem no atendimento aos pacientes em pós-operatório, encontraram-se os valores médio de 28,7 (DP $\pm 6,0$ ), mínimo de 25,7 e máximo de 42,5. Ou seja, em média cada paciente requereu $28,7 \%$ do tempo da equipe de enfermagem ou 7 horas e 28 minutos. Os cuidados prestados pela equipe de enfermagem foram mais requeridos por pacientes que apresentaram hipotensão $(p=0,003)$ e arritmia $(\mathrm{p}=0,000)$ no pós-operatório.

\section{DISCUSSÃO}

A literatura científica aponta que os principais fatores de risco para o surgimento de alterações no período pós-operatório de cirurgias cardíacas se associam à idade do paciente, ao sexo, à hipertensão arterial, a diabetes, a níveis elevados de colesterol lipoproteína de baixa densidade (LDL) e baixos de colesterol lipoproteína de alta densidade (HDL), ao tabagismo, à obesidade, ao sedentarismo, à história familiar ${ }^{2}$, ao tipo de medicação utilizada no pré-operatório e a fatores de risco intraoperatórios (tipo de cirurgia, tempo de permanência em CEC, tempo de permanência em internação e uso de medicação específica) ${ }^{9}$. No entanto, no presente estudo, as principais complicações identificadas no POI foram de origem cardíaca ou hematológica, e apenas o fator de risco não modificável histórico familiar de cardiopatias apresentou correlação estatística com complicação cardiológica, e o fator de risco modificável dislipidemia, com o diagnóstico de enfermagem integridade de pele prejudicada.

No tocante à hipertensão arterial sistêmica (HAS), ela é tida como o principal fator de risco para doença arterial coronariana e marcadamente relacionada à hereditariedade ${ }^{10,11} \mathrm{e}$ ao óbito em pacientes submetidos à $\mathrm{RM}^{3}$. Contudo, apesar de a HAS ter sido o antecedente pessoal mais recorrente no presente estudo, não foi verificada correlação estatística com óbito e complicações pós-operatórias.

No momento atual, a RM apresenta índices de sobrevida de $97 \%$ em um ano e de $81 \%$ após dez anos de pós-operatório $^{12}$. Tal prognóstico pode ser atribuído à técnica empregada para o pinçamento da aorta e ao tempo de $\mathrm{CEC}^{12}$.

Apesar disso, acredita-se que o uso da CEC induz o organismo a uma resposta inflamatória sistêmica, causada pela circulação do sangue por meio do circuito de extracorpórea e pela formação de microêmbolos ${ }^{13}$. No entanto, o mecanismo fisiopatológico das lesões que ocorrem nos órgãos após a CEC permanece pouco esclarecido ${ }^{14}$.
O uso de CEC pode levar a complicações, entre elas: disfunção renal, infarto agudo do miocárdio, comprometimento neurológico, disfunção cognitiva, fibrilação atrial e disfunção ventricular ${ }^{11}$, risco de acidente vascular cerebral intraoperatório associado à manipulação da aorta ascendente e arritmias, além de estar relacionado ao risco de hemorragia no período pós-operatório, a baixo débito cardíaco, a derrame pleural, a tamponamento cardíaco e à coagulopatia ${ }^{2,10}$.

Estudo prospectivo analisou os prontuários de 72 pacientes submetidos à RM e identificou que o maior número de complicações esteve ligado a distúrbio hidroeletrolítico (61\%), acidobásico (35\%), glicêmico (32\%), cardíaco (13\%) e pulmonar $(10 \%)$. As demais complicações foram de origem renal $(7 \%)$, neurológica (6\%), infecciosa (3\%) e hepática $(1 \%)^{11}$.

Outra análise desenvolvida com 145 pacientes adultos submetidos à cirurgia cardíaca (45\% RM, 26\% cirurgia valvar, $18 \%$ cirurgia combinada e $11 \%$ outros tipos de operação cardíaca) apontou que as principais complicações pós-operatórias nas primeiras 24 horas incluíram perda de sangue (58\%), disfunção pulmonar (34\%), arritmias (6\%) e necessidade de reintubação $(3 \%)^{15}$.

Em contrapartida, o presente estudo identificou como principais complicações as de origem hematológica, representadas por sangramento e instabilidade hemodinâmica, seguidas das de origem do sistema cardiológico, como arritmias, hipotensão, fibrilação atrial e síndrome do baixo débito cardíaco. Identificou-se, também, que a ingestão insuficiente de nutrientes se apresenta como fator de risco para incidência de angina no POI de RM.

A baixa pressão diastólica aumenta o risco de choque, uma vez que a perfusão insuficiente induz à hipóxia celular e tissular e poderá, eventualmente, promover falência de órgãos e sistemas. Salienta-se, também, que o risco de queda é aumentado em pacientes com hipotensão ortostática ${ }^{16}$.

Outro aspecto que merece destaque no pós-operatório de cirurgia cardíaca é o fato de que os pacientes permanecem com a mobilidade física prejudicada por períodos prolongados, dificultando a ação dos músculos da panturrilha e, por conseguinte, prejudicando o retorno venoso. Além disso, os agentes anestésicos interferem na vasodilatação e na constrição normal, reduzindo a perfusão para as proeminências ósseas e para as regiões sob pressão. Assim, o diagnóstico de enfermagem integridade de pele prejudicada é frequente e pode ser resultado de pressão intensa e/ ou prolongada, em combinação com o cisalhamento, resultado de ato mecânico como a própria incisão cirúrgica e de fatores como microclima, nutrição, perfusão, comorbidades e condição clínica ${ }^{17,18}$. 
Portanto, em função da mudança metabólica causada nas cirurgias de $\mathrm{RM}^{14}$, pacientes que realizam o procedimento e que possuem histórico familiar de cardiopatias e comorbidades, como dislipidemia, possuem mais chances de desenvolver complicações cardiológicas e apresentar lesão cutânea, aumentando a demanda de trabalho da equipe de enfermagem.

Os diagnósticos de enfermagem indicaram associações estatisticamente significantes com diversas variáveis clínicas e relacionadas ao procedimento anestésico-cirúrgico. Dessa forma, essa complexidade atribuída à assistência dos pacientes submetidos a cirurgias cardíacas requer cuidados especiais em todo o perioperatório, destacando-se o período pós-operatório.

Atualmente, nas UTI, o grau de dependência do paciente, a complexidade da doença, as características da instituição, os processos de trabalho, a planta física e o perfil dos profissionais da equipe determinam a carga de trabalho de enfermagem ${ }^{19}$. Estimar a demanda de cuidados faz-se necessária para assegurar a qualidade da assistência e subsidiar a quantificação de pessoal nas diferentes unidades hospitalares, visto que uma equipe superdimensionada implica alto custo e uma equipe reduzida pode determinar a queda da qualidade de atendimento dos pacientes ${ }^{7}$.

Logo, existem instrumentos que colaboram nessa avaliação, entre eles o therapeutic intervention scoring system-28 (TISS-28) e o NAS. Para desenvolvimento do presente estudo, o indicador escolhido foi o NAS. Apesar de o TISS-28 possuir extensa abordagem na literatura científica, a aplicação prática mostrou falhas estruturais para a medida total da carga de trabalho de enfermagem, uma vez que as atividades concernentes ao cuidado indireto do paciente, como tarefas organizacionais, não estavam incluídas em sua composição ${ }^{7}$.
O NAS, por sua vez, tem apresentado crescente uso em UTI e leva em conta atividades básicas, suporte ventilatório, suporte cardiovascular, suporte renal, suporte neurológico, suporte metabólico e intervenções específicas. Neste estudo, identificou-se que, em média, cada paciente requereu $28,7 \%$ do tempo da equipe de enfermagem ou 7 horas e 28 minutos.

Contudo, acredita-se que esse número possa estar subestimado, dada a característica associada à coleta retrospectiva de informações, que pode ter sido prejudicada por ausência de registros adequados. Assim, para melhor estimativa, os profissionais devem ser orientados sobre o funcionamento do instrumento para que as atividades sejam anotadas de maneira correta. No futuro, novos estudos serão capazes de determinar, de forma mais precisa, a carga de trabalho associada ao cuidado pós-operatório de pacientes submetidos a cirurgias cardíacas.

\section{CONCLUSÃO}

No presente estudo, obteve-se que as principais complicações pós-operatórias foram cardiológicas e estiveram relacionadas ao histórico familiar de cardiopatia. O tempo de CEC esteve associado ao aparecimento de fibrilação atrial no POI, aos diagnósticos de enfermagem risco de sangramento e ao déficit para o autocuidado.

Por fim, o diagnóstico de enfermagem mais frequente foi o risco de infecção, e os pacientes que apresentaram hipotensão $(\mathrm{p}=0,003)$ e arritmia $(\mathrm{p}=0,000)$ no pós-operatório foram os que necessitaram de mais quantidade de cuidados pela equipe de enfermagem no pós-operatório.

\section{REFERÊNCIAS}

1. Nascimento LS, Dias RM, Dutra CDT, Silva LMC, Pedrosa LN, Araújo JS, et al. Fatores de risco para doenças crônicas não transmissíveis e variáveis sociodemográficas de servidores públicos. Rev Bras Promo Saúde [lnternet]. 2015 [acessado em 3 mar. 2018];28(2):230-9. Disponível em: http://periodicos.unifor.br/RBPS/article/view/3570/pdf

2. Colósimo FC, Sousa AG, Silva GS, Piotto RF, Pierin AMG. Hipertensão arterial e fatores associados em pessoas submetidas à cirurgia de revascularização do miocárdio. Rev Esc Enferm USP. 2015;49(2):200-1. http://dx.doi.org/10.1590/S0080-623420150000200003

3. Koerich C, Lanzoni GMM, Erdmann AL. Factors associated with mortality in patients undergoing coronary artery bypass grafting. Rev Latino-Am Enfermagem. 2016;24:1-9. https://doi. org/10.1590/1518-8345.0708.2748

4. Carvalho ARS, Matsuda LM, Carvalho MSS, Almeida RMSSA Schneider DSLG. Complicações no pós-operatório de revascularização miocárdica. Ciênc Cuid Saúde [Internet]. 2006 [acessado em 21 mar. 2018];5(1):50-9. Disponível em: https://pesquisa.bvsalud.org/portal/ resource/pt/bde-12303

5. Torrati FG, Dantas RAS. Extracorporeal circulation and complications during the immediate postoperative period for cardiac surgery. Acta Paul Enferm. 2012;25(3):340-5. http://dx.doi.org/10.1590/ S0103-21002012000300004 
6. Brasil. Conselho Nacional de Saúde. Resolução n 466, de 12 de dezembro de 2012. Aprova as diretrizes e normas regulamentadoras de pesquisas envolvendo seres humanos [Internet]. Brasília, 2012 [acessado em 5 abr. 2018]. Disponível em: https://conselho.saude. gov.br/resolucoes/2012/Reso466.pdf

7. Queijo AF, Padilha KG. Nursing Activities Score (NAS): adaptação transcultural e validação para a língua portuguesa. Rev Esc Enferm USP. 2009;43(Núm. Esp.):1-8. http://dx.doi.org/10.1590/ S0080-62342009000500004

8. North American Nursing Diagnosis Association. Diagnósticos de enfermagem da NANDA; definições e classificação 2011-2014. Porto Alegre: North American Nursing Diagnosis Association; 2013.

9. Soares GMT, Ferreira DCS, Gonçalves MPC, Alves TGSA, David FL, Henriques KMC, et al. Prevalência das principais complicações pósoperatórias em cirurgias cardíacas. Rev Bras Cardiologia [Internet]. 2011 [acessado em 7 maio 2018];24(3):139-46. Disponível em: http://sociedades.cardiol.br/socerj/revista/2011_03/a_2011_v24_ n03_01 prevalencia.pdf

10. Quadros FF, Gutierrez LLP, Ribeiro JL. Obesidade e fatores de risco cardiovascular em filhos de obesos: uma revisão. Scientia Medica [Internet]. 2013 [acessado em 8 abr. 2018];23(2):119-26. Disponível em: http://revistaseletronicas.pucrs.br/ojs/index.php/scientiamedica/ article/viewFile/11675/9653

11. Oliveira JLM, Hirata MH, Sousa AGMR, Gabriel FS, Hirata TDC, Tavares IS, et al. Sexo masculino e hipertensão arterial são preditores de placa à angiotomografia das coronárias. Arq Bras Cardiol [Internet]. 2015 [acessado em 10 mar. 2018];104(5):409-16. Disponível em: http:// www.scielo.br/pdf/abc/v104n5/pt_0066-782X-abc-20150028.pdf http://doi.org/10.5935/abc.20150028

12. Sobral MLP, Santos Jr. SF, Sá JC, Terrazas AS, Trompieri DFM, Sousa TAN, et al. Aperfeiçoamento em técnica de perfusão cardioplégica no pinçamento único de aorta: resultados iniciais. Rev Bras Cir Cardiovasc [Internet]. 2014 [acessado em 10 mar. 2018];29(2):229-34. Disponível em: http://www.scielo.br/pdf/rbccv/v29n2/0102-7638-rbccv-29-02-0229.pdf http://doi.org/10.5935/1678-9741.20140026
13. Henriques LS, Forte WC. Alterações imunológicas pós circulação extracorpórea. Rev Bras Alerg Imunopatol [Internet]. 2000 [acessado em 12 jun. 2018];23(4):143-150. Disponível em: http://www.sbai.org. $\mathrm{br} /$ revistas/Vol234/alt.htm

14. Christensen MC, Krapf S, Kempel A, Heyman CV. Costs of excessive postoperative hemorrhage in cardiac surgery. J Thorac Cardiovasc Surg [Internet]. 2009 [acessado em 13 abr. 2018];138(3):687-93. Disponível em: https://www.jtcvs.org/article/S0022-5223(09)00324-9/ fulltext https://doi.org/10.1016/j.jtcvs.2009.02.021

15. Oliveira JMA, Silva AMF, Cardoso AB, Lima FF, Zierer MS, Carvalho ML. Complicações no pós-operatório de cirurgia cardiovascular com circulação extracorpórea. Rev Interd [Internet]. 2015 [acessado em 14 maio 2018];8(1):9-15. Disponível em: https://revistainterdisciplinar. uninovafapi.edu.br/index.php/revinter/article/view/584/pdf_177

16. Kapadohos T, Angelopoulos E, Vasileiadis I, Nanas S, Kotanidou A, Karabinis A, et al. Determinants of prolonged intensive care unit stay in patients after cardiac surgery: a prospective observational study. J Thorac Dis [Internet]. 2017 [acessado em 2 abr. 2018];9(1):70-9. Disponível em: https://www.ncbi.nlm.nih.gov/pmc/articles/ PMC5303108/

https://dx.doi.org/10.21037\%2Fjtd.2017.01.18

17. Associação Brasileira de Estomaterapia. Classificação das lesões por pressão. Consenso NPUAP 2016 [Internet]. Associação Brasileira de Estomaterapia; 2016 [acessado em 21 abr. 2018]. Disponível em: http://www.sobest.org.br/textod/35

18. Rocha LA, Maia TF, Silva LF. Diagnósticos de enfermagem em pacientes submetidos à cirurgia cardíaca. Rev Bras Enferm. 2006;59(3):321-6. http://dx.doi.org/10.1590/S0034-71672006000300013

19. Altafin JAM, Grion CMC, Tanita MT, Festti J, Cardoso LTQ, Veiga CFF, et al. Nursing Activities Score e carga de trabalho em unidade de terapia intensiva de hospital universitário. Rev Bras Ter Intensiva [Internet]. 2014 [acessado em 12 mar. 2018];26(3):292-8. Disponivel em: http:// www.scielo.br/pdf/rbti/v26n3/0103-507X-rbti-26-03-0292.pdf http://dx.doi.org/10.5935/0103-507X.20140041 